\title{
Linx
}

Revue des linguistes de l'université Paris X Nanterre

$75 \mid 2017$

Imaginaires de la ponctuation. Ordre et inquiétude du discours

S'inscrire singulièrement dans la langue.

L'imaginaire de la ponctuation dans les écrits de poilus ordinaires et dans les textes d'écrivains combattants

\section{Anne-Laure Kiviniemi}

\section{OpenEdition \\ Journals}

Édition électronique

URL : http://journals.openedition.org/linx/1926

DOI : 10.4000/linx.1926

ISSN : 2118-9692

Éditeur

Presses universitaires de Paris Nanterre

Édition imprimée

Date de publication : 22 décembre 2017

Pagination : 161-179

ISBN : 978-2-84016-308-4

ISSN : 0246-8743

\section{Référence électronique}

Anne-Laure Kiviniemi, « S'inscrire singulièrement dans la langue. L'imaginaire de la ponctuation dans les écrits de poilus ordinaires et dans les textes d'écrivains combattants », Linx [En ligne], 75 | 2017, mis en ligne le 23 novembre 2018, consulté le 01 mai 2019. URL : http://journals.openedition.org/ linx/1926 ; DOl : 10.4000/linx.1926

Ce document a été généré automatiquement le 1 mai 2019.

Département de Sciences du langage, Université Paris Ouest 


\title{
S'inscrire singulièrement dans la langue. L'imaginaire de la ponctuation dans les écrits de poilus ordinaires et dans les textes d'écrivains combattants
}

\author{
Anne-Laure Kiviniemi
}

«La ponctuation [...] pourrait bien engager plus qu'elle n'en a l'air » : cette remarque de Dürrenmatt (2000:3) pousse à envisager les marques de ponctuation comme autant de traces de la subjectivité du scripteur. La ponctuation reste «l'apanage des personnes, sans emprise complète des codes et des normes » (Favriaud, 2011: 7) ${ }^{1}$. Elle constitue donc un angle pertinent pour étudier le processus de stylisation, entendue par Jaubert (2014: 68) comme «la création d'une valeur qui accompagne l'appropriation de la langue en discours " par une subjectivité :

Le sujet parlant s'approprie la langue et cette appropriation engendre progressivement une émergence du style, perçu comme une valeur. Dans sa genèse, cette valeur peut être envisagée sous de multiples saisies, de l'acception minimale de l'expressivité dans le discours, à l'idée d'une 'forme singulière'. (Jaubert, 2007 : 250)

2 Tout locuteur manifesterait ainsi à des degrés variables sa singularité et son conventionnalisme - et par là même son imaginaire langagier - à travers la ponctuation. Il s'agira ici de mettre à l'épreuve la théorie de la progressivité de l'émergence du style en étudiant l'usage qui est fait de la ponctuation dans des écrits de scripteurs plus ou moins habiles. Cet usage leur permet-il de s'inscrire singulièrement dans la langue ? Que révèlet-il de leur sentiment à l'égard de la ponctuation? Pour montrer que les ressorts qui actionnent le style se trouvent cachés dans les formes les plus banales de la langue seront confrontés des usages d'écrivains et d'écrivants à des étapes différentes de la diagonale du style (Jaubert, 2007, 2012, 2014) : la déponctuation apollinairienne sera mise en regard 
de l'absence de ponctuation des textes d'un peu-lettré ; à la ponctuation émotive de Céline sera opposée la ponctuation rationalisante d'un aspirant brillant.

\section{La progressivité de l'émergence du style}

3 L'évolution stylistique est tantôt imputée aux grands écrivains,

La singularité de l'écrivain est interprétée comme la variation différentielle qu'intégrera l'évolution collective. S'il a d'abord parlé seul, c'est qu'il parlait d'avance au nom de tous... (Starobinsky dans Spitzer, $1970: 19$ )

tantôt portée au crédit de certains groupes sociaux :

Parce qu'ils sont directement confrontés à une réalité jusqu'alors impensable, les soldats sont amenés [...] à essayer de nommer, à leur manière, ce qui échappe à la langue standard comme à la langue littéraire, de relater, avec leurs propres mots, leur expérience, leurs émotions, et leur parole offre parfois des expressions qui [...] révèlent dans leur extrême simplicité, une profondeur et une efficacité perceptibles bien au-delà du monde du front. Aussi certains écrivains semblent voir dans la parole populaire une source de renouvellement possible de la langue littéraire. (Mariot, $2013: 83$ )

Le moteur de l'évolution stylistique est-il donc littéraire ou ordinaire? Pour Jaubert, l'émergence du style est progressive. Elle rejoint les positions du linguiste Gustave Guillaume, selon lequel il y a continuité dans l'investissement de la langue en discours. Parler est une activité de transition lors de laquelle le locuteur exploite le contenu de la langue (le dicible) en vue de construire un discours (le dit). Le passage de la langue au discours est ainsi assuré par le mécanisme dynamique qu'est l'acte de langage. La langue, entendue comme un savoir-dire permettant au locuteur de se représenter et d'exprimer n'importe quelle pensée, est formée d'institué - qui tend du côté normatif - et de permissions de variation. Les permissions normatives (de simple expression) soustendent l'emploi momentané d'un fait de langue en discours; les permissions extranormatives sont comprises comme des permissions d'expressivité (Guillaume, 1997 : 206). Tout sujet parlant dans l'élaboration d'un discours recourt, dans des proportions variables, à l'institué, aux permissions de simple expression et aux permissions d'expressivité, sachant qu'aucune phrase n'est dépourvue d'expressivité : « appropriation de la langue et valeur, si minime soit-elle ne peuvent se délier» (Jaubert, $2014: 198$ ). L'actualisation de la langue en discours s'accompagne donc d'une stylisation plus ou moins marquée, c'est-à-dire d'une subjectivisation et d'une esthétisation variables.

6 Le style est ainsi appréhendé comme un objet évolutif (plus ou moins subjectif et plus ou moins esthétisé) selon la valeur - expressive, distinctive ou d'individualisation - que le sujet parlant confère au discours. L'émergence du style peut être décrite sous forme de diagonale en trois étapes (correspondant aux trois emplois du mot style: un style, du style, le style) - où la prise de valeur croissante du discours se fait en naviguant entre singularité (pôle particularisant) et conventionnalisme (pôle universalisant). Dans un premier temps, le style est envisagé comme la valeur expressive dont se charge un discours élaboré dans le cadre d'un échange langagier spécifique : «le style fonctionne [alors] comme un révélateur social, la marque de l'appartenance à un groupe, à une école, etc., dont il s'agit de maîtriser les codes " (Jaubert, $2014: 68$ ). Dans un deuxième temps, il est la valeur distinctive dont un discours, qui se conforme à des patrons génériques et discursifs, se dote: le locuteur emprunte alors le style propre à un projet 
communicationnel. Dans un troisième temps, le style se conçoit comme valeur d'individualisation : il se singularise pour devenir une forme individuée.

7 Dans cette théorisation, le style se déclare progressivement dans la congruence $d u$ discours à différentes visées (Jaubert, 2012: 197). Nous nous proposons de vérifier la validité de cette conception en analysant l'usage de faits de langue ponctuatifs à des places différentes sur la diagonale du style. Par son omniprésence et sa plasticité, la ponctuation, constitue un angle pertinent pour étudier le processus de stylisation.

\section{La ponctuation au croisement de l'usage normatif et de l'usage singulier}

8 La ponctuation peut être considérée d'un côté comme " une norme - les règles de ponctuation - où tout écart est une faute à corriger, de l'autre un art où tout écart est ou peut être - style » (Serça, $2004: 15)$.

9 La norme telle qu'elle apparaît dans les manuels d'école primaire républicaine est conforme à la tradition grammaticale selon laquelle la ponctuation participe et de l'interprétation des textes et de leur oralisation. Les préceptes inscrits dans la Nouvelle grammaire française de Noël et Chapsal $(1845: 203)$ - «La ponctuation sert à marquer la distinction des sens, et les pauses qu'on doit faire en lisant » - ont été retenus dans la grammaire scolaire de Larive et Fleury $(1910: 5-6)$ :

La ponctuation est employée pour éclaircir le sens des phrases et pour indiquer les pauses que l'on doit faire en lisant.

[...] On emploie la virgule après les noms, les adjectifs, les pronoms, les verbes, etc., qui entrent dans une énumération; avant et après les parties de phrase qui ne sont pas indispensables au sens. [...] On emploie le point à la fin des phrases, quand le sens est entièrement fini.

10 L'école républicaine se charge donc d'inculquer un usage normatif de la ponctuation, réduite à sa fonction grammaticale de garante de clarté de l'écrit. Or, on ne peut faire abstraction du fait que tout scripteur entretient «un rapport tout personnel et éminemment subjectif avec les signes de ponctuation » (Serça, $2004: 15$ ).

11 C'est le cas de poètes comme Mallarmé ou Apollinaire qui, à la même époque, font de la ponctuation un outil de stylisation, donc de subjectivisation du discours. Au-delà de sa fonction logique, la ponctuation peut être appréhendée comme marque d'un style personnel - elle « traduit concrètement et plus encore peut-être que les mots prononcés [l'affectivité du sujet]» (Latin, 1988: 96) — puisqu'elle a aussi pour rôles de « révéler d'autres modes de structuration que ceux officialisés par la grammaire dogmatique, faire évoluer les représentations officialisées de la syntaxe » (Favriaud, $2011: 7$ ).

12 Voyons maintenant comment l'imaginaire de la ponctuation de scripteurs contemporains ${ }^{2}$ d'habileté inégale se manifeste dans leurs écrits. Considèrent-ils la ponctuation comme une norme à appliquer ou plutôt comme un espace d'expressivité et un lieu de subjectivisation du discours? 


\section{Ponctuation superflue versus déponctuation signifiante}

13 La ponctuation dans la correspondance de guerre de Baptiste Lapouge est infinitésimale. L'échange épistolier de 16019 mots ne compte que 88 points, 1 virgule, 1 deux-points et 1 parenthèse. Cela n'empêche pas la ponctuation d'être «facilement restituable, ce qui montre que la structure syntaxique de l'écrit est maîtrisée » (Klippi \& Kiviniemi, 2015). Tout se passe comme si le scripteur considérait que la ponctuation n'est pas indispensable à la bonne intelligence de ses lettres. La syntaxe suffit à délivrer un message intelligible. Lapouge ne semble pas avoir conscience du fait que l'absence de ponctuation peut générer des difficultés de lecture, comme dans l'extrait (1) où l'absence de point après «Planche » prête, dans un premier temps, à confusion:

(1) (Lapouge_23.02.1916)

nous avons un cantonement les cheveaux sont dans des baraques en Planche dans les greniers des maisons abondoner nous ne sommes pas exposer dans le Village que nous sommes est jamais tomber un obus

ou des effets stylistiques :

(2) (Lapouge_17.02.1915)

Si je peux rester ici quelques mois de plus une foi au beaux jours la campagne seras moin mauvaise et encore autre chose je pourais mas Suzette peut-ettre te avoir plus souvent <souvent> car comme je tais dejas dit ces quelques heures que jai passer auppres de toi ça ne me sufit pas ça me fait ennuyer davantage jai pas eut le temp ma cherie de te prouver combien je $t$ aime. mai tu le sais bien que je tadore par dessue tout et que je voudrais comme autrefois revivre auppres de toi nous ettion et nous serion encore si heureux de pouvoir nous partager nos soufrance et nos joie par des inombrables Baisers te dire mas Suzanne je taime bien

Que veut tu mas cherie faut se resigner conserver notre courage et cette Maudite campagne terminer pour toujour je serais aupres de toi je ne te quiterais plus car $j$ aurais trop soufert de ton absence

15 La carence de ponctuation, qui oblige à lire la séquence quasiment d'un seul souffle, donne une impression de jaillissement émotif irrépressible. Les deux exemples mentionnés ci-dessus sont marginaux: dans la grande majorité des écrits de Lapouge, l'élaboration d'un texte logique et rationnel rend la ponctuation superflue.

C'est le même sentiment de superflu qui semble animer la démarche d'Apollinaire (2014 :

62-63) :

Pour ce qui concerne la ponctuation, je ne l'ai supprimée que parce qu'elle m'a paru inutile et elle l'est en effet, le rythme même et la coupe des vers, voilà la véritable ponctuation et il n'en est point besoin d'une autre.

17 Ainsi Le cinquième poème secret adressé à Madeleine Pagès ${ }^{3}$ (Apollinaire, 2005 : 457-461) est composé d'une première partie en vers et d'une seconde partie consistant en une suite de calligrammes. Dans la première partie, les séquences textuelles sont séparées par des sauts de lignes, chacune commence par une majuscule ; dans la seconde partie, ce sont les calligrammes qui se font chacun porteur d'une séquence textuelle. Ces séquences, mises en valeur par la ponctuation blanche (soit l'ensemble des blancs qui donnent au texte sa mise en forme), unifient en ensembles sémantiques ce que la ponctuation noire (soit l'ensemble des signes graphiques qui opèrent au niveau de la phrase) diviserait, séparerait, associerait et hiérarchiserait. Le poète organiserait donc ses productions, non 
sur le modèle phrastique, mais sur un modèle séquentiel pour une nouvelle structuration du texte.

Cette structuration séquentielle du texte se retrouve chez Lapouge, qui ne se conforme pas, lui non plus, aux enseignements de l'école primaire. Il ne se sert pas de la ponctuation pour découper ses phrases, mais a recours - avec parcimonie - à des points ou à des sauts de lignes pour séparer des segments textuels. La ponctuation n'est pas utilisée de manière phrastique mais s'inscrit dans la dimension textuelle et se manifeste essentiellement aux démarcations des parties de la lettre selon l'ars dictaminis ${ }^{4}$ et lors des changements énonciatifs (voir Annexe 2.). Cette pratique témoignerait donc d'un imaginaire où la fonction sémantique de la ponctuation l'emporterait sur sa fonction syntaxique.

Sur les 87 points recensés dans la correspondance Lapouge, $92 \%$ se trouvent en milieu de paragraphe. Jamais Lapouge ne termine une lettre par un point final. Le peu-lettré a donc recours à la ponctuation noire et à la ponctuation blanche de façon alternative pour matérialiser les articulations de son discours. La ponctuation blanche détermine des paragraphes, unités sémantiques elles-mêmes, s'il le faut, segmentées en unités de signification plus petites matérialisées par des points.

Là où Lapouge respecte les conventions épistolaires en matière d'ordonnancement, Apollinaire bouleverse la composition :

L'invention, surtout poétique, doit commencer par l'expérience d'un manque, celui du sens et de la cohérence [...]. La suppression de la ponctuation [noire] tend donc à faire l'expérience de la perte d'un cadre logique et rationnel, d'une partie même de la grammaire en tant qu'elle devrait être exhibée dans la phrase. Pour le poète, c'est libérer 1'image de la rhétorique : les images produiront un effet d'autant plus fort qu'elles auront été imprévisibles à la logique. [...] Le poète brouille l'armature logique pour contraindre le lecteur à s'abandonner à la dérive poétique. (Fraisse, $2007: 190-193)$

L'abandon de la logique permet l'apparition d'images se succédant au rythme des séquences matérialisées par la ponctuation blanche et les calligrammes. Mais cet abandon de la logique n'est qu'apparent: «à la logique rationnelle se substitue une logique affective plus secrète» (id. 191) parce que nécessitant la recomposition des liens implicites dont est tramé le texte.

Un même phénomène, l'absence de ponctuation, peut ainsi être appréhendé aux deux extrêmes de la diagonale du style : fait d'expressivité chez Lapouge - ce qui ne l'empêche pas d'être parfois susceptible d'interprétations stylistiques - il devient chez Apollinaire fait de style qui, mis en convergence avec la perte de la métrique traditionnelle, la perte de la raison sous les images et la perte de l'ordre chronologique révèle la forme sousjacente (voir Cahné, 2005) qu'est l'esthétique de la perte (voir Fraisse, 2007).

23 Les démarches des deux hommes sont différentes: l'un s'attache à mettre en texte un discours - la ponctuation met en évidence les articulations du texte; l'autre s'attache à mettre en œuvre son texte - la déponctuation noire garantit l'effet poétique.

\section{Ponctuation rationnelle versus ponctuation émotive}

Déponctuer, c'est complexifier. Si la fonction essentielle de la ponctuation semble bien être la clarification du discours, les usages qui vont maintenant être abordés font état de rôles autres que le seul éclaircissement du message. 


\subsection{La ponctuation pour cacher l'émotion}

lit pas dans la ponctuation. Dans cette lettre ne figure qu'un seul point d'exclamation. On recense par contre 21 points-virgules, ce qui semblerait témoigner d'un imaginaire linguistique profondément marqué par la norme de l'époque. Sa correspondance illustrerait cette conception de la ponctuation essentiellement grammaticale et syntaxique adoptée au $19^{\mathrm{e}}$ siècle : "La ponctuation est l'anatomie du langage ou de la pensée [...] : ponctuer c'est disséquer les phrases » (Le Journal de la typographie et de la lithographie, 1876, cité par Lorenceau, 1980 : 52).

$\mathrm{Au}$ niveau phrastique, le point-virgule a une fonction de segmentation. La grammaire scolaire de Larive et Fleury (1910: 5-6) préconise d'employer «le point-virgule pour séparer les principales divisions d'une même phrase, notamment quand on a déjà fait usage de la virgule pour les subdivisions. Ex. Les groseilles, les raisins, sont des baies ; les cerises, les prunes, sont des fruits à noyau ».

Toutefois, à y regarder de plus près, l'emploi que Fugier fait ici du point-virgule, semble aller au-delà des recommandations primaires de séparation des divisions d'une même phrase. En reliant, à l'intérieur de la phrase complexe, des structures syntaxiquement complètes, le point-virgule met en évidence leur (inter)dépendance sémantique : les propositions en cascade se précisent les unes les autres. Les points-virgules servent à matérialiser les liens logiques entre sous-phrases - consécution des arguments :

(3) (Fugier_03.08.1916)

Ici, comme je vous l'ai expliqué 100 fois, il n'y a absolument rien à faire ; [pointvirgule de conséquence : donc] papa s'est bien trompé quand, dans sa lettre au $C^{\text {ne }}$, il dit que les asp ${ }^{\text {ts }}$ de la classe 14 n'ont pas de valeur $\mathrm{m}^{\text {re }}$; [point-virgule de cause : car] ils en ont beaucoup, au contraire, ayant 2 ans de guerre et sont ou plutôt devraient être très appréciés ; [point-virgule de coordination] et même avant eux il $\mathrm{y}$ a des asp ${ }^{\text {ts }}$ qui ont 2 ans d'active et sont chefs de $\mathrm{S}^{\text {on }}$ depuis 20 mois !

(4) (Fugier_03.08.1916)

J'ai soigneusement observé la ligne boche tous ces jours ci ; j'ai repéré un créneau où l'on voit très bien la tête des Boches tous les matins; $\mathrm{j}$ 'ai déplacé des sacs à terre de façon à laisser un petit intervalle, j'ai pointé un fusil, l'ai fixé avec des pieux; le matin, un homme observe à la jumelle dans le périscope et, quand il voit la tête de Fritz, il me prévient ; je tire ; comme j'emploie des balles perforantes qui trouent les boucliers, il y a des chances pour que le Boche reçoive, même si la balle manque le trou du bouclier, ce qui est peu probable. 

justifier une position. Fugier tente de juguler son ressenti par un texte bien construit et une position bien argumentée. L'imaginaire mis en œuvre ici serait celui d'une ponctuation envisagée comme lieu de subjectivisation du discours : matérialisant les liens logiques, elle oriente le lecteur pour le contraindre à se ranger au jugement du scripteur. La cohésion phrastique sert la cohérence textuelle.

Alors qu'une ponctuation exclamative ferait étalage de l'emportement du jeune homme, la ponctuation choisie exhibe ses efforts de tempérance et de modération. La ponctuation, chargée de rationaliser le discours, atténue du même coup la charge émotive du texte: elle produit comme un « effet de sourdine " (Spitzer, 1970: 209) et confère au texte une impression de retenue contrebalançant le caractère vindicatif des reproches énoncés. L'auteur de la lettre peut alors défendre ses vues sans fâcher ses destinataires, exprimer son courroux tout en faisant preuve de maîtrise et ainsi convaincre de sa maturité. Ce dernier objectif transparaît encore en fin de lettre, lorsque Fugier se met en scène comme guerrier ayant le droit de vie ou de mort sur d'autres hommes - bien loin de l'image d'assisté requérant une recommandation parentale pour pouvoir progresser que lui ont renvoyé ses correspondants.

À cet usage singulier de la ponctuation comme moyen de canaliser l'émotion, il peut être intéressant en contrepoint, d'opposer la pratique d'un contemporain qui lui confère le rôle inverse : chez Céline, elle est chargée de porter la vindicte de l'écrivain.

\subsection{La ponctuation pour exhiber l'émotion}

L'écrivain s'en sert pour «resensibiliser la langue, qu'elle palpite plus qu'elle ne raisonne » (Céline, 1969 : 137) :

Mes trois points sont indispensables !... indispensables, bordel Dieu !... je le répète :

indispensables à mon métro [émotif] ! me comprenez-vous Colonel ? (Céline, 2007

[1955] : 52)

Alors que chez Fugier la ponctuation exhibe la logique et cache l'émotion, chez Céline la ponctuation substitue l'émotion à la logique. L'écrivain rejette la phrase grammaticale par l'accumulation des points de suspension. Le texte n'est « plus fait que de courtes séquences de mots, chacune portée par une émotion » (Godard, 2009 : XXIV). Les troispoints, marqueurs de phrases incomplètes, actualisent, non des phrases bouclées, mais des séquences. L'incomplétude phrastique, parce qu'elle se réitère via le recours massif aux points de suspension, devient au niveau textuel marque de dislocation du phrasé. Les trois-points en kyrielle mettent en évidence " un découpage de la phrase qui ne respecte pas les frontières syntaxiques » (Serça, $2012:$ 226-227).

À l'opposé de Fugier, qui revient après le point-virgule sur la première structure pour la préciser ou pour la justifier, Céline désoriente son lecteur après les points de suspension par une structure imprévisible : «la pause des trois-points brise la continuité d'une phrase qui, après une première structure complète syntaxiquement, se poursuit de façon imprévisible" (Watine, 2014 : 311-312). La transgression des normes ponctuatives et l'usage inédit des points de suspension permet à Céline de subvertir la langue classique et par là-même d'éviter la platitude du discours.

36 Fait d'expressivité chez Fugier, le point-virgule, rationalisant et conciliant, sert un objectif pragmatique immédiat: convaincre sans concession et sans virulence. La 
ponctuation se doit d'amener les deux parties à un consensus. Fait de style chez Céline, les points de suspension sollicités à l'extrême, servent la vision subversive célinienne. Ce dernier adopte « une écriture qui doit donner l'illusion du langage parlé et en restituer l'émotion directe' " pour « renverser les conventions d'une langue écrite, jugée froide, rationnelle et, par là-même, mensongère parce que liée à une vision conformiste et rassurante de la réalité en général, et de la société en particulier» (Latin, 1988: 33). La ponctuation déstructurante vise à désorienter pour provoquer une remise en question de l'ordre établi (linguistique et sociétal).

\section{Conclusion}

L'usage et la transgression des codes de ponctuation manifestent de positionnements individuels et originaux face à un ensemble de normes et ce quelle que soit l'habileté rédactionnelle des scripteurs dans le corpus envisagé. Tous, et pas seulement «les écrivains [,] créent leur propre ponctuation, partie prenante de leur style » (Serça, 2012 : 71).

Les pratiques ponctuatives susmentionnées, dans leur forme (l'absence de ponctuation) ou dans la fonction qui leur est allouée (cacher ou montrer l'émotion), montrent qu'il y a bien continuum entre écriture ordinaire et écriture littéraire et soutiennent la position de Bally :

La pénétration du langage et de la sensibilité n'est pas propre à l'expression littéraire seulement, c'est la marque de tout langage spontané; l'écrivain se contente de transposer à son usage les thèmes qu'il trouve dans le langage de tout le monde et de les faire servir à ses fins, qui sont esthétiques et individuelles, tandis que le langage de tous est actif et social. La tâche de la stylistique [...] est précisément, tout en se confinant à la langue commune, de mettre à nu les germes du style, de montrer que les ressorts qui l'actionnent se trouvent cachés dans les formes les plus banales de la langue. Style et stylistique sont deux domaines à la fois distincts et voisins : tout signe expressif de la langue pose cette question: dans quelles conditions un type expressif employé par tout le monde peut-il se transformer en un procédé littéraire, reconnaissable à ces deux caractères : intention esthétique et marque individuelle. (Bally, 1952 : 60-61)

ou celle de Genette, chez qui le style est présenté comme une propriété générale des discours (voir Jenny, 1993 : 114).

Si les usages ici analysés, dans leur originalité, décrivent bien quatre inscriptions singulières dans la langue, on pourrait opposer des scripteurs ordinaires qui mettent la ponctuation à profit pour faire valoir articulations et liens logiques, orientant ainsi le lecteur dans son interprétation du texte, à des auteurs qui, eux, cherchent à le déstabiliser. Plus valable toutefois apparaît la divergence d'intentionnalité discernée entre le tenant d'une ponctuation visant à la clarté du texte (Lapouge) et les partisans d'une ponctuation visant à l'efficacité de l'écrit. Tant Fugier que Céline et Apollinaire usent d'une ponctuation fonctionnelle mise à profit à des fins précises. La ponctuation rationalisante du jeune aspirant atténue la charge émotive du texte tandis que les usages des deux hommes de lettres entraînant perte du cadre logique et rationnel, de la cohérence et de la prévisibilité illustrent les obsessions respectives des deux hommes de lettres - la perte pour Apollinaire, la subversion pour Céline - thématiques que ces écrivains combattants ne pouvaient transmettre avec la langue d'avant-guerre. 


\section{BIBLIOGRAPHIE}

FUGIER, A. 1915-1918. Correspondance, journal de guerre, carnet de campagne, dans Fonds Fugier, Historial de Péronne.

LAPOUGE, B. 1908-1919. Correspondance, dans Fonds Lapouge, Centre d'Études Edmond Michelet, Brive.

APOLLINAIRE, G., 2005, Lettres à Madeleine, Paris, Gallimard.

APOLLINAIRE, G., 2014, Alcools. Poèmes 1898-1913, Le livre de poche, édition numérique.

BALLY, C., 1965 [1952], Le langage et la vie, Genève, Librairie Droz.

CAHNÉ, P., 2005, « Linguistique et critique littéraire : psycho-mécanique et analyse stylistique », Langue française, 147, p. 121-126.

CÉLINE, L.-F., 2007 [1955], Entretien avec le Professeur Y, Paris, Gallimard, <http://archive.org/ details/EntretiensAvecLeProfesseurY>

CÉLINE, L.-F., 2009, Lettres, Paris, Gallimard.

DÜRRENMATT, J., 2000, « Avant-Propos », La Licorne, 52, <http://licorne.edel.univ-poitiers.fr/ document5835.phps.

FAVRIAUD, M., 2011, « Approches nouvelles de la ponctuation diachroniques et synchroniques ». Langue française, 172, p. 3-18.

FRAISSE, L., 2007, « Apollinaire et l'esthétique de la perte », Studia Romanica Posnaniensia, Poznan, Adam Mickiewicz University Press, p. 187-200.

GUILLAUME, G., 1997, Leçons de linguistique de Gustave Guillaume 1946-1947 et 1947-1948, série A, Esquisse d'une grammaire descriptive de la langue française $(V)$ et Esquisse d'une grammaire descriptive de la langue française (VI), publiées par le Fonds Gustave Guillaume de l'Université Laval sous la direction de Valin, R., Hirtle, W. et Lowe, R. Québec - Paris, Presses de l'Université Laval Klincksieck.

HINDUS, M., 1969, L.-F. Céline tel que je l'ai vu, Paris, L'Herne.

JAUBERT, A., 2007, « La diagonale du style. Étapes d'une appropriation de la langue », Pratiques, 135-136, p. 47-62.

JAUBERT, A., 2012, « Pour une théorie intégrative du style », dans C. Narjoux (éd). Au-delà des frontières : Perspectives de la stylistique contemporaine, Frankfurt am Main, Peter Lang, p. 197-208.

JAUBERT, A., 2014, « L'avènement du style », dans L. Himy-Piéry, J-F. Castille et L. Bougault, (dir.), Le style, découpeur de réel. Faits de langue, effets de style, Rennes, Presses universitaires de Rennes, p. 67-74.

JENNY, L., 1993, « L'objet singulier de la linguistique », Littérature, 89, p. 113-124.

KIVINIEMI, A-L., 2013, « L'écriture des poilus à l'aune des normes scolaires - une étude stylistique ", Cahiers AFLS On-Line, 18.1, p. 4-45, http://afls.net/cahiers/18.1/Kiviniemi.pdf. 
KLIPPI, C. \& KIVINIEMI, A.-L., 2015, « L'écriture de deux frères d'armes, déshérités du français Une caricature de la langue nationale? », dans K. Jeppesen Kragh et J. Lindschouw (éds), Les variations diasystématiques et leurs interdépendances dans les langues romanes, Strasbourg, ELiPhi.

LARIVE \& FLEURY, 1876, Les Exercices français de première année, Paris, Armand Colin.

LARIVE \& FLEURY, 1910, La deuxième année de grammaire. Orthographe, rédaction, littérature, Paris, Armand Colin. $125^{\mathrm{e}}$ édition.

LATIN, D., 1988, Le Voyage au bout de la nuit de Céline : roman de la subversion et subversion du roman, Bruxelles, Palais des Académies.

LECLAIR, L. \& ROUZÉ, C., 1874, Grammaire française, Paris, Belin.

MARIOT, N., 2013, Tous unis dans la tranchée? 1914-1918, les intellectuels rencontrent le peuple, Paris, Seuil.

NOËL \& CHAPSAL, 1845, Nouvelle grammaire française, Paris, Maire-Nyon, Roret, Hachette, Delalain, $38^{\mathrm{e}}$ édition.

PONTIS, P.-C. 1881. Petite grammaire de la ponctuation, Paris, Hetzel.

RICQUIER, L. 1873, Traité de ponctuation précédé d'un précis d'analyse logique, Paris, Librairie de L'Écho de la Sorbonne.

SERÇA, I., 2004, « La ponctuation : petit tour d'horizon », L'Information Grammaticale, 102, 11-17.

SERÇA, I., 2012, Esthétique de la ponctuation, Paris, Gallimard.

SPITZER, L., 1970, Études de style, Paris, Gallimard.

WATINE, M.-A., 2014, « Prévisibilité phrastique et style parlé chez Céline », dans L. Himy-Piéry, JF. Castille et L. Bougault, (dir.), Le style, découpeur de réel. Faits de langue, effets de style, Presses universitaires de Rennes, p. 303-313.

\section{ANNEXES}

Annexe 1. Lettre du 23 mars 1915 de Baptiste Lapouge à sa femme ${ }^{5}$ 


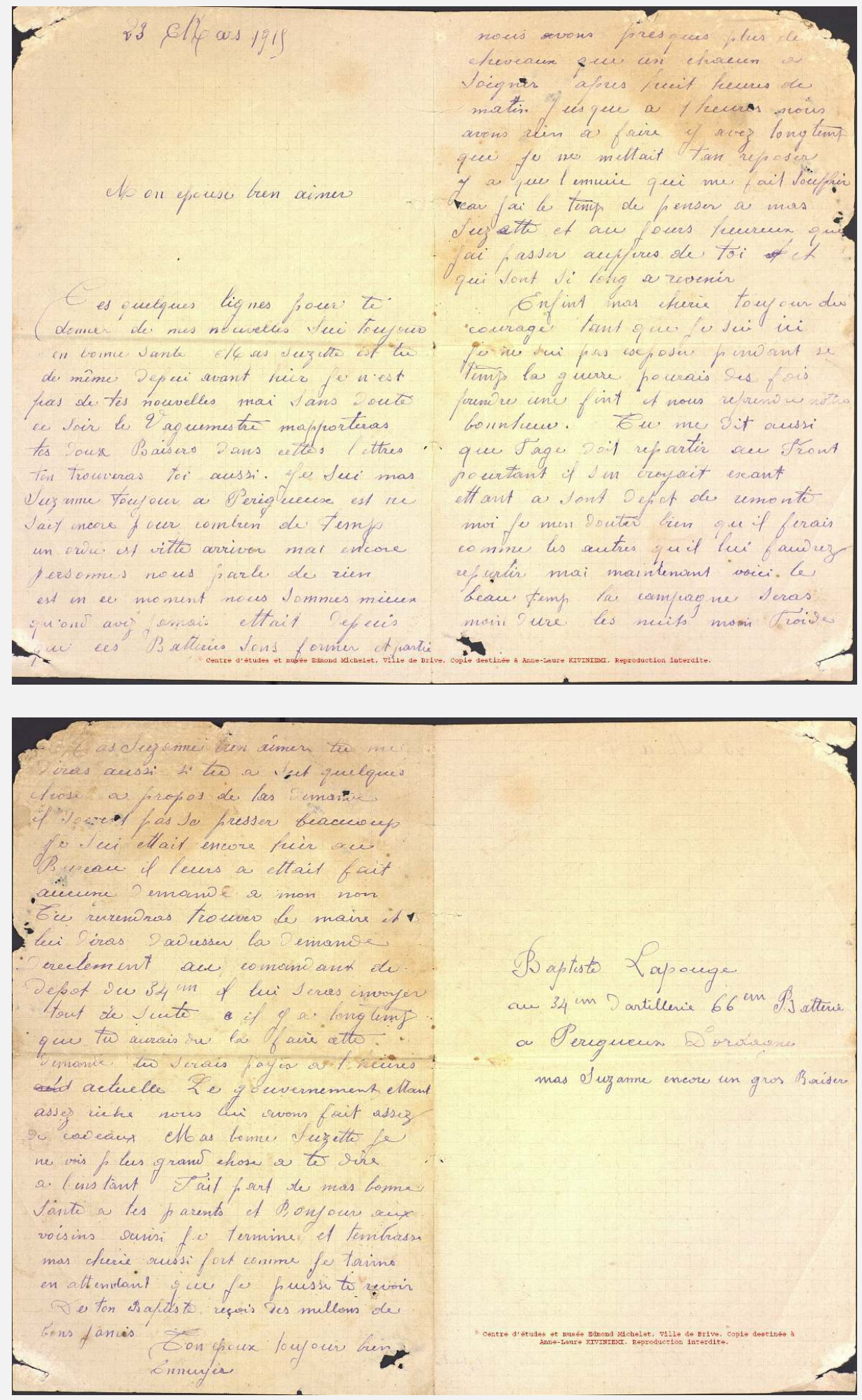

Annexe 2. Ponctuation textuelle dans la lettre du 23 mars 1915 de Baptiste Lapouge 

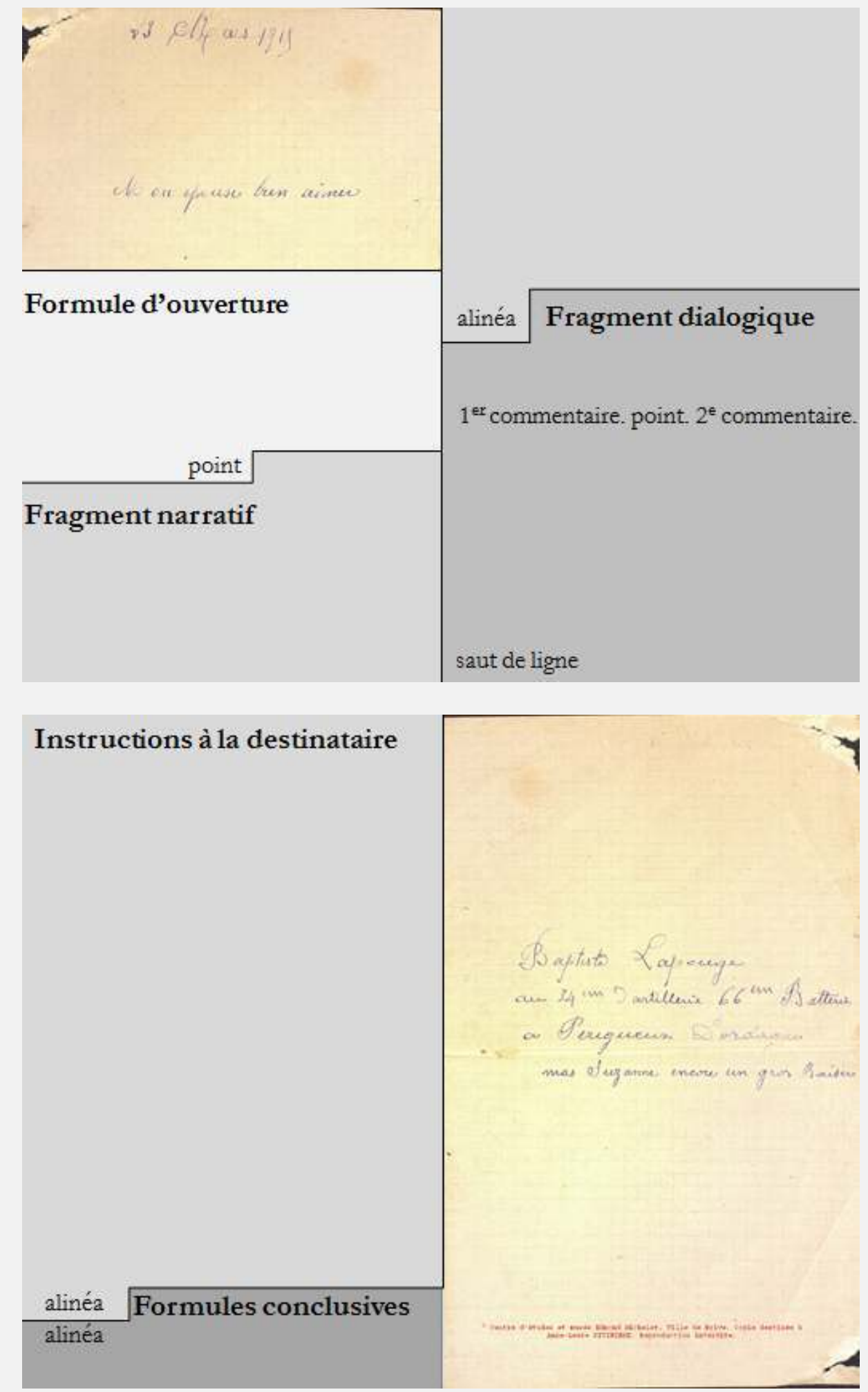


\section{NOTES}

1. Fin $19^{\mathrm{e}}$ - début $20^{\mathrm{e}}$ siècle, l'usage normatif de la ponctuation est largement diffusé par les traités de ponctuation (Ricquier, 1873 ; Pontis, 1881) et les grammaires scolaires (Noël et Chapsal, 1845 ; Leclair et Rouzé, 1874 ; Larive et Fleury, 1910).

2. Apollinaire (1880-1918), Lapouge (1885-1973), Céline (1894-1961), Fugier (1896 - 1966).

3. Laurence Campa (Apollinaire, 2005 : 157) estime que ce poème a été écrit entre la mi-octobre et le début novembre 1915.

4. La composition épistolaire fait partie des savoirs enseignés à l'école primaire : les manuels scolaires, que ce soit en instruction civique (Viala : 1896: 257-259) ou en langue française, y consacrent une place notable. La série des Larive et Fleury en témoigne : la Deuxième année de Grammaire explique les principes de la composition épistolaire et fournit des modèles de lettres (1910 : 180, 202-205) et les Exercices français de première année (et de deuxième année) proposent de nombreux « sujets de lettres » et insistent sur le respect de la forme : «Les élèves feront bien de donner aux exercices qui suivent la forme d'une lettre véritable dans laquelle figureront l'entête, la signature et l'adresse.» (1876:119-121).

5. Coll. Centre d'études et musée Edmond-Michelet, ville de Brive (Corrèze-France). Cotes : 1 CEM 42 (lettre du 23.03.1915).

\section{RÉSUMÉS}

La ponctuation, par son omniprésence et sa plasticité, constitue un angle pertinent pour étudier le processus de stylisation. Il s'agira ici de mettre à l'épreuve la théorie de la progressivité de l'émergence du style en étudiant l'usage qui est fait de la ponctuation dans des écrits de scripteurs plus ou moins habiles. Que révèle-t-il de leur imaginaire linguistique et leur permet-il de s'inscrire singulièrement dans la langue?

The ubiquity and plasticity of punctuation make it a relevant perspective to consider when exploring the process of stylization. The aim of this article is to test the theory of a progressive emergence of style by studying the use of punctuation in the writings of more or less skilful writers. How does their usage of punctuation allow them to mark their individuality through language and what does it reveal of their linguistic imaginary?

\section{INDEX}

Mots-clés : ponctuation à valeur expressive, ponctuation à valeur sémantique, écriture épistolaire, guerre de 1914-1918 
AUTEUR

ANNE-LAURE KIVINIEMI

Université de Tampere 